\title{
Attenuation of Cross-Flow Fan Noise Using Porous Stabilizers
}

\author{
Huanxin Lai, Meng Wang, Chuye Yun, and Jin Yao \\ Key Lab of Safety Science for Pressurized Systems, School of Mechanical and Power Engineering,
} East China University of Science and Technology, Shanghai 200237, China

Correspondence should be addressed to Huanxin Lai, hlai@ecust.edu.cn

Received 1 March 2011; Revised 15 July 2011; Accepted 31 July 2011

Academic Editor: M. Razi Nalim

Copyright () 2011 Huanxin Lai et al. This is an open access article distributed under the Creative Commons Attribution License, which permits unrestricted use, distribution, and reproduction in any medium, provided the original work is properly cited.

This paper presents a qualitative analysis of controlling the cross-flow fan noise by using porous stabilizers. The stabilizer was originally a folded plate. It is changed into a porous structure which has a plenum chamber and vent holes on the front wall. In order to investigate the influences of using the porous stabilizers, experiments are carried out to measure the cross-flow fan aerodynamic performances and sound radiation. Meanwhile, the internal flow field of the fan is numerically simulated. The results show that the porous stabilizers have not produced considerable effect on the cross-flow fan's performance curve, but the noise radiated from the fan is strongly affected. This indicates the feasibility of controlling the cross-flow fan noise by using the porous stabilizers with selected porosity.

\section{Introduction}

In order to improve the nowaday indoor environment for daily life and work, air-conditioners are becoming widely used [1]. Because cross-flow fans (CFFs) generally have large capacities of mass flow and are compact in size [2-5], they are often used to move air in the air conditioners. However, a CFF is also well known for the complex flow configuration, such as the irregular geometry of the impeller and the stabilizer. As a result, the internal flows of CFF are very complex, while the eccentric vortex is a typical phenomenon [6]. An illustration of the internal flow-field of a CFF is shown in Figure 1. These complexities have made it difficult to form a universal designing theory for CFF, and therefore, the flow efficiencies of the CFF are relatively poor [7-9], as compared with axial or centrifugal fans.

Noise radiation from CFF is a very important issue of concern for indoor environment. In order to attenuate the noise radiation from the CFF, Koo et al. [7] and Tsai et al. [10] used skewed stabilizers and skewed rotors, respectively. Because the spanwise size of a CFF is generally several times larger than the radial size, it is possible to skew the impeller and/or the stabilizer so as to avoid the blades at different axial locations pass the stabilizer simultaneously. On the other hand, Moon et al. [4] tried to modulate the blade passing frequency noise by using random-pitch impellers. All these considerations are aimed at weakening the vortical flow impingements on the stabilizer. Indeed, some effects in cutting down the noise radiation have been achieved by using these methods.

Porous media are widely used in sound controlling. Generally, porous materials are applied to wrapping up the sound sources so as to dissipate the sound energy into heat. Different from this usage, it is also possible to use porous materials to control flows. An example is the porous inserts for improving the aerodynamic performance of a supercritical airfoil by weakening the shock/boundary layer interaction [11]. Similarly, Wilcox [12] experimentally explored to control cavity flow and sound radiation by replacing the cavity floor with a layer of a porous medium. Lai and Luo [13], Jeng and Payne [14], and Kim and Chokani [15] numerically investigated the flow fields of cavities with porous walls. In their numerical models, the normal velocity over porous surface was approximated by the linear form of Darcy's pressure-velocity law. Both experimental and numerical results revealed that the porous wall arrangement had dramatically changed the flow pattern, which was believed to be related to the pressure redistribution inside the cavities. In another example, Hughes and Dowling [16] used perforated linings with a rigid surface placed behind 


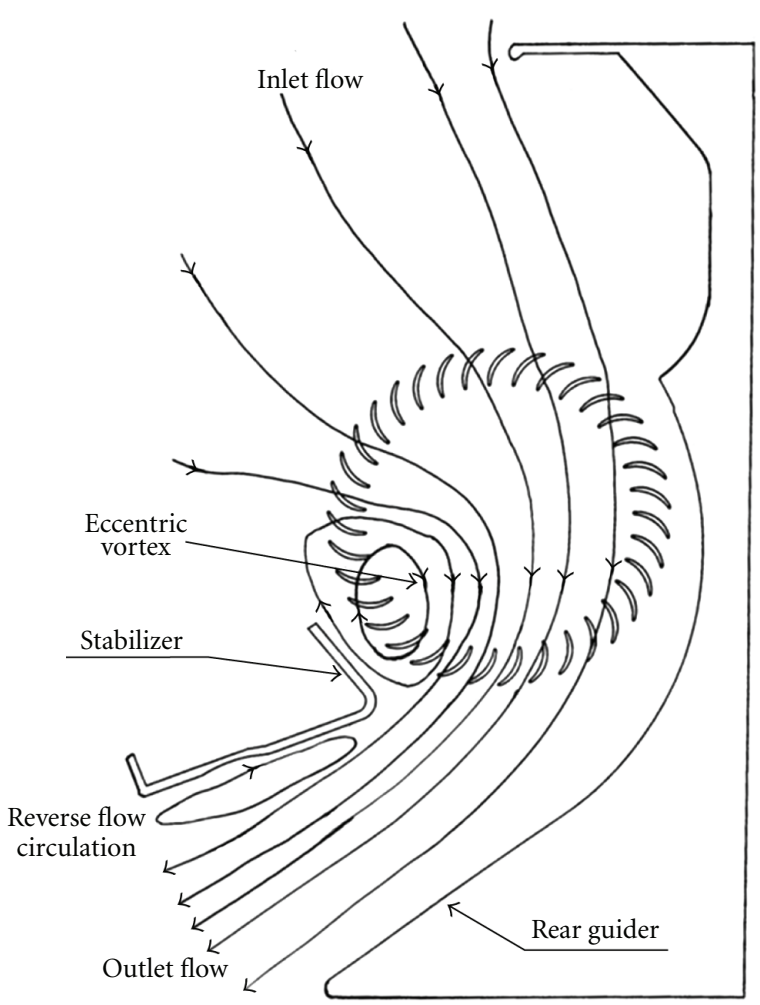

Figure 1: Typical flow pattern in a cross-flow fan.

for jet flow controlling. The sound-absorbing capability over a range of frequencies was proved by both theory and experiment. In these cases, the flow through the porous surface or the perforated liners plays a crucial role. For the purpose of sound controlling, these techniques are different from the wrapping-up of sound sources, which merely dissipate high-frequency sound energy into heat. The employment of porous media can not only damp sound energy but also change the flow itself, which is the source of sound. It thus has the promise of controlling low-frequency sound as well as high-frequency sound [13].

This paper presents a qualitative experimental and numerical study of noise controlling for a CFF by using porous structures. The eccentric vortex is the largest vortical motion in a CFF $[1,4,5]$, and its location is in the vicinity of the stabilizer, where flow/structure interaction such as vortical flow impingement on the wall occurs. Therefore, the stabilizer is considered for using porous structure in this paper. As shown in Figure 2, the stabilizer of the CFF is originally a folded plate, but it is changed into a porous structure in our study. A third wall is added to the stabilizer fold so as to form a triangular chamber. Vent holes were drilled in the front wall. Compared with other soundcontrolling strategies for the CFF, such as those mentioned in above, using porous stabilizers will not change the geometry of the flow configuration. To the authors' knowledge, this strategy of using porous stabilizers for CFF noise control has not been attempted by researchers. Based on these backgrounds, this paper qualitatively explore the feasibility of attenuating the noise of CFF using porous stabilizers.
TABLE 1: Specifications of the studied CFF.

Number of blades 35

External diameter of the impeller, $D_{2}$

$94 \mathrm{~mm}$

Internal diameter of the impeller, $D_{1}$

$70 \mathrm{~mm}$

Impeller axial length, $L$

$580 \mathrm{~mm}$

External blade angle

$26^{\circ}$

Internal blade angle

$90^{\circ}$

Experiments and numerical simulations are carried out to find the qualitative effects of this modification on the aerodynamic performance and noise of the CFF.

\section{Experimental Setup}

2.1. The Tested Fan. The tested fan is the indoor part of a split-type air conditioner. Its geometry is shown in Figure 3 and Table 1. In order to simplify the problem so as to focus on our sound-controlling purpose, the fins of the heat exchanger and the air-return grill have been removed from the tested fan.

2.2. Aerodynamic Test. The test rig for fan performance, shown in Figure 4, was built according to the Chinese GB/T1236-2000 Standard on Industrial Fans Test Methods and Acceptance Condition (equivalent to ISO 5801:1997). The outflow from the tested fan is discharged into the free space, while the suction side is connected to a plenum chamber, whose upstream is the inflow Venturi nozzle for flow rate measurement. At downstream of the Venturi nozzle, an auxiliary fan is employed to overcome the pressure losses generated by the flow passage. A honeycomb straightener duct is placed in between of the auxiliary fan and the plenum chamber so as to reduce the nonuniform disturbance to the inflow of the tested fan. Pressure measurements in the plenum chamber and the Venturi nozzle are performed using slanted manometers, which result in a $1 / 10 \mathrm{~mm}$ water accuracy.

The tested fan is driven by an AC-motor which is provided with regulated voltage supply. The rotational speed of the fan is monitored and measured by a photoelectric sensor, and it is found to vary in a range of $\pm 1 \%$ at the testing rotational speed $n=1258 \mathrm{rpm}$.

2.3. Acoustic Measurement. The acoustic measurements are carried out in an anechoic chamber, which is built according to the ISO3745 standard and has a net volume of $4.5 \mathrm{~m} \times$ $4.2 \mathrm{~m} \times 2.9 \mathrm{~m}$. The ambient noise and the cutoff frequency of the anechoic chamber are $20 \mathrm{~dB}$ and $100 \mathrm{~Hz}$, respectively. The sound pressure signal is measured by an MP205-9293 piezoelectric sensor connected to an amplifier and a signal conditioner, according to the experimental method regulated by the ISO 5151:1994 Standard. The sensitivity of the sensor is $50 \mathrm{mV} / \mathrm{Pa}$. The sound pressure signal is sampled at the frequency of $40 \mathrm{kHz}$ and is analyzed using fast Fourier transform (FFT) algorithm with the A-weighting filter applied in Hanning window. The number of samples for each 


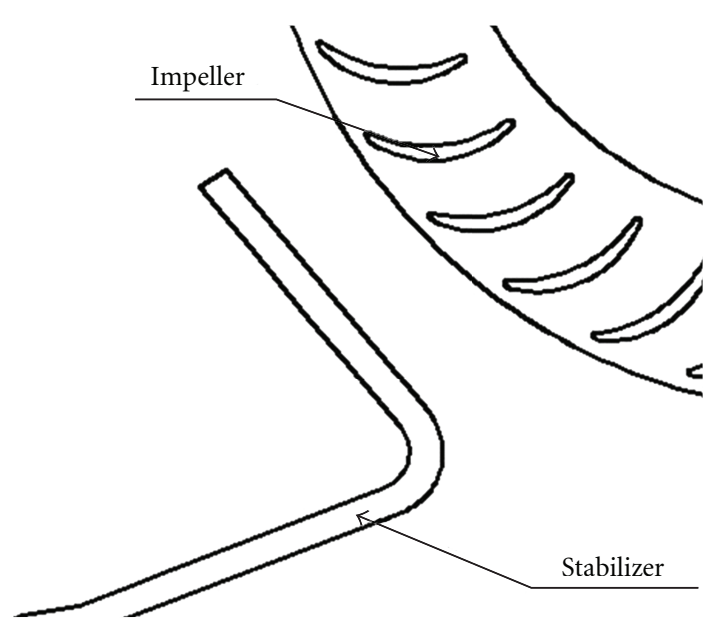

(a)

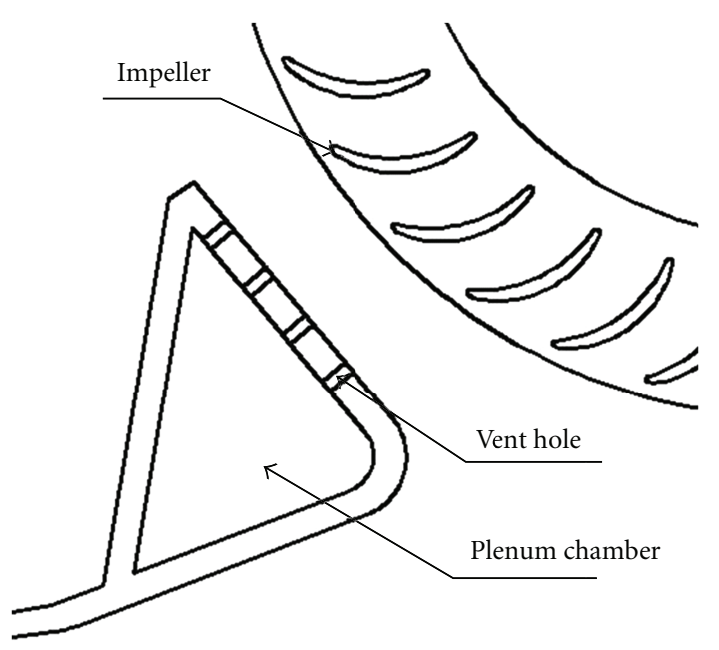

(b)

FIGURE 2: Stabilizer of cross-flow fan. (a) The original folded-plate stabilizer. (b) The porous stabilizer with plenum and vent holes.

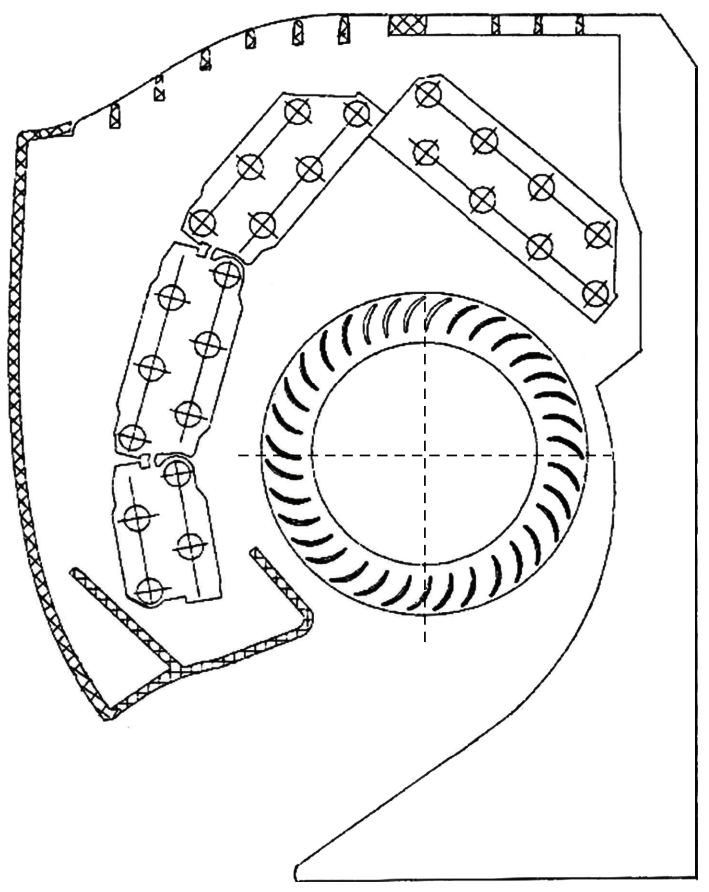

(a)

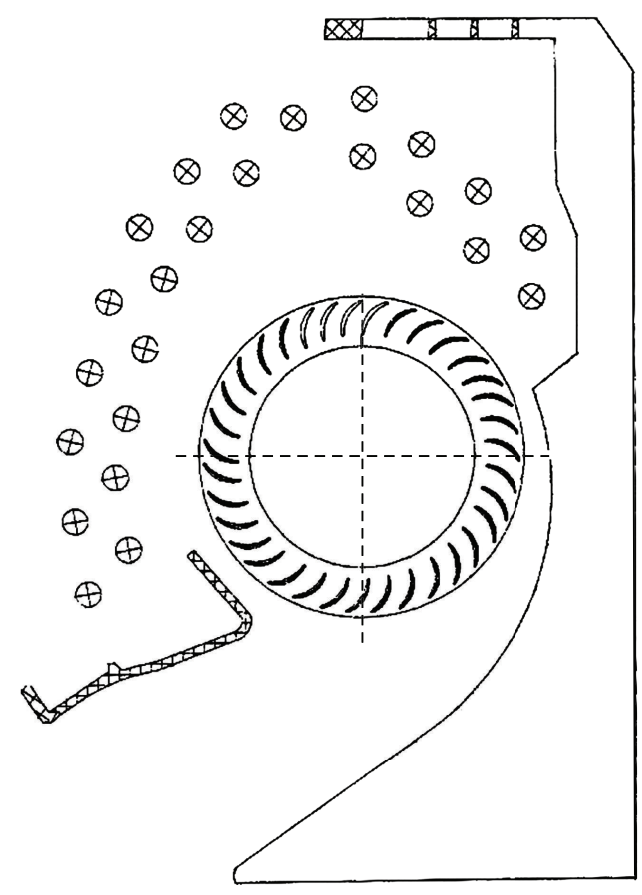

(b)

Figure 3: Geometry of the cross-flow fan from a split-type air-conditioner. (a) With air-return grill and heat exchanger. (b) Without airreturn grill and heat exchanger.

window is 4096. The spectra of the noise signal are produced using the average of eight blocks with $50 \%$ overlapping.

\section{Numerical Method}

The commercial CFD software package FLUENT [17] is employed for calculating the internal flow of the CFF. The Reynolds number based on the inflow velocity and the impeller diameter is 11000 , while the Mach number at the fan outlet is approximately only 0.017 . The flow is, therefore, regarded as incompressible. The governing equations include the conservation laws of the mass and momentum. For the closure of turbulence, the so-called standard $k-\varepsilon$ turbulence model [18] is employed. Readers may have realized the existence of flow transition in the plenum chamber of the stabilizer, which means the standard $k-\varepsilon$ turbulence model 


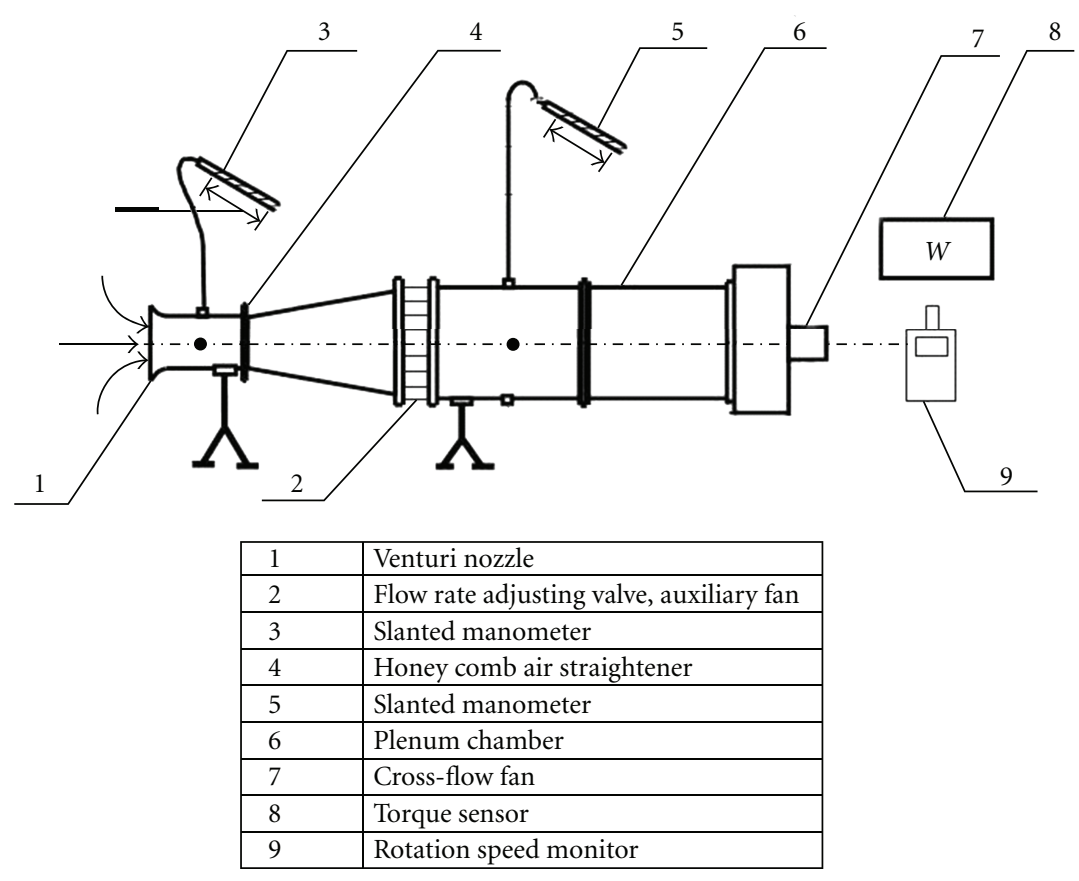

FIGURE 4: Schematic of the test rig used to measure fan performance.

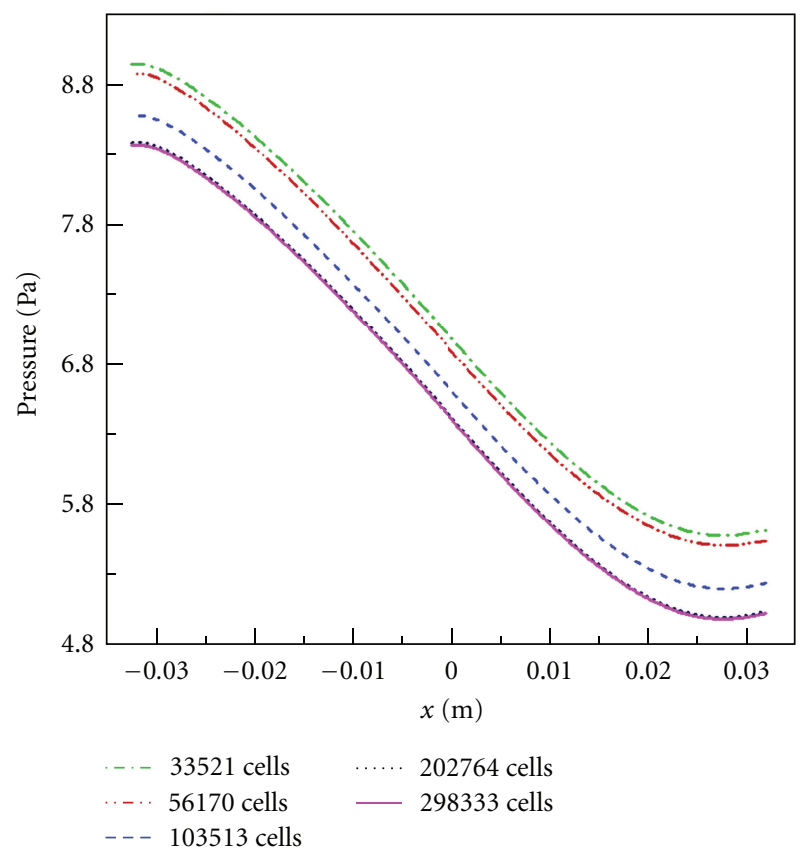

Figure 5: Profiles of static pressure calculated using five meshes. Plotted along $y=0$, in the range of $-0.32 \leq x \leq 0.32$.

for fully turbulent flow is not very suitable. However, this paper is only for a qualitative analysis while the standard $k$ $\varepsilon$ turbulence model is one of the most classical and is well established, so it is selected.

The spanwise length of the CFF impeller is more than 6 times of the diameter, while the flow is almost uniform in the spanwise direction, so the flow crossing the impeller perpendicularly is often treated as two dimensional. Such a two-dimensional treatment is widely adopted by researchers $[1,4,5,9]$ and is also applied in this paper. The computational domain includes the zone inside the fan and the porous stabilizer. And it is extended at the inlet and outlet bounds, following the experiences of Moon et al. [4], Toffolo et al. [9] and Shih et al. [1]. The boundary conditions include the no-slip condition at the walls, the velocity inflow condition at the fan entrance, and the pressure outflow condition. The calculation employs the pressure-based SIMPLE algorithm [19]. The convection term is discretized by a second-order upwind scheme, while the diffusion is calculated using central difference. In order to calculate the unsteady flow caused by the impeller rotation in the fan, a sliding interface is employed to separate the impeller and the outer zone surrounding it. The initial fields for unsteady calculation are given by the steady calculation using the multi-reference frame (MRF) method in the FLUENT package. Grid-dependency check is carried out in the MRF calculation by refining the mesh step by step. Five meshes with 33521, 56170, 103513, 202764, and 298333 triangular cells, respectively, are tested. The static pressure along the linesection $y=0$ in the range of $-0.32 \leq x \leq$ 0.32 , which passes the impeller center point horizontally, is plotted in Figure 5. The profiles vary with the meshes, but the variance is diminishing when the mesh is refined. When the mesh is refined to have 202764 cells, the result has almost negligible plotting difference with that from the mesh of 298333 cells. So, the mesh with 202764 cells can be regarded as fine enough to give the grid-independ solution, and it is selected for unsteady calculation. The zonal mesh near 


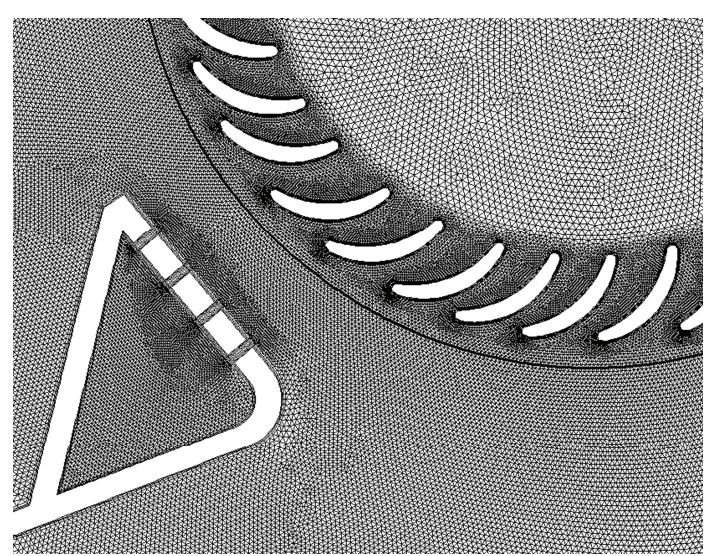

FIGURE 6: The zonal grids including the porous stabilizer.

the stabilizer is shown in Figure 6. The unsteady calculation starts with the initial fields and is carried out according to a time-marching strategy. Because implicit temporal method is employed, the marching time step for unsteady calculation can be theoretically very large. However, a too large time step will damage the temporal resolution for unsteady calculation [19]. In this paper, the marching time step is decided according to the rotation speed of impeller and the number of circumferential grid nodes at the sliding interface. Our purpose is to make the grids at the interface slide only one node circumferentially per time step so that the meshes at both sides of the sliding interface are always matched. According to this designation, the time step is set to be $1 / 500$ of the period of the impeller rotation in this paper, because the mesh employed has 500 uniformly spaced grid nodes along the sliding interface. Periodical unsteady solution is achieved after marching time equal to about 4 to 5 rotations of the impeller.

\section{Results}

The CFF with its original folded-plate stabilizer (depicted as Case A) and four porous stabilizers is considered. The four porous stabilizers have 2000 vent holes of $0.3,0.5,1.0$, and $1.5 \mathrm{~mm}$ in diameter, respectively, drilled in the front wall in rows $5 \mathrm{~mm}$ apart. And therefore, the porosities (defined as the total area of the vent holes to the area of the front wall) for the stabilizers are $0.855 \%, 2.375 \%, 9.5 \%$, and $21.37 \%$, respectively. For the convenience of description, the CFFs using these porous stabilizers are denoted by Case B, C, D, and $\mathrm{E}$, respectively.

In the above five cases, the stabilizer shape of Case A is different from with the other cases beside the holes. In order to examine the influence of such difference, a new stabilizer depicted as Case A1 is considered for comparison. The shape of stabilizer of Case Al is the same with the porous stabilizers, but it has no vent hole in the front plate. Figure 7 compares the contours of static pressure at an instant, while Figure 8 compares the time history of pressure oscillation at a monitored point. There is almost no plotting difference between Case A and Case A1 in the results. These
TABLE 2: Overall sound pressure level of the tested CFF (dBA).

\begin{tabular}{lcccc}
\hline Case A & Case B & Case C & Case D & Case E \\
\hline 46.0 & 44.2 & 44.0 & 47.5 & 47.0 \\
\hline
\end{tabular}

comparisons confirm that Case A1 is not necessary and therefore dropped in the further experimental and numerical analysis.

4.1. Performance Curve and Sound Signal. Figure 9 is the fan's $\psi-\varphi$ curve measured at the rotation speed of $n=1258 \mathrm{rpm}$, where the nondimensional flow rate coefficient $\varphi$ and the pressure coefficient $\psi$, respectively, are defined as

$$
\begin{gathered}
\varphi=\frac{Q}{\left(L D_{2} U_{2}\right)}, \\
\psi=\frac{2 \Delta p}{\rho U_{2}^{2}},
\end{gathered}
$$

$Q$ and $\Delta p$ are the flow rate $\left(\mathrm{m}^{3} / \mathrm{s}\right)$ and the static pressure rise $(\mathrm{Pa})$ of the fan, respectively; $U_{2}$ is the velocity $(\mathrm{m} / \mathrm{s})$ of the blade at the external edge of the impeller. For the case of using the original stabilizer (Case A), the curve profile is quite smooth. Compared with this, the measured points for the cases of using porous stabilizers (Cases B to E) exhibit some wiggles, but they are basically uniformly distributed in both sides of the Case A. Moreover, although the porosity increases steadily from Case B to Case E, it has not caused a visible tendency of shifting in the $\psi-\varphi$ curves. Therefore, the wiggles in Figure 9 are more likely caused by possible uncertainty and/or error of the measurement rather than caused by using the porous stabilizers.

The $1 / 3$ octave band spectra of noise from the tested CFF for different stabilizers, measured at rotation speed $n=$ $1258 \mathrm{rpm}$ and at the maximum flow rates for all cases, are shown and compared in Figure 10. The spectra for the five cases have similar distributions, they are basically broadband spectra. The energy-containing noise modes are in the frequency range of from $100 \mathrm{~Hz}$ to $10000 \mathrm{~Hz}$, with the peaks at around $1000 \mathrm{~Hz}$. The overall sound pressure level (SPL) of the fan is shown in Table 2. It can be seen that the SPL values for the Case B and Case $\mathrm{C}$ are lower than the original stabilizer case (Case A) by 1.8 and $2 \mathrm{dBA}$, respectively. Case $\mathrm{D}$ and Case E, however, have SPL higher than Case A; the SPL is increased by 1.5 and $1 \mathrm{dBA}$, respectively. The results in Table 2 indicate the possible existence of an optimal porosity for the stabilizers so as to silence the fan to the lowest SPL value. But apparently, more stabilizers with different porosities should be tested if we need find out such a case experimentally.

4.2. Flow Fields. As we have mentioned in the introduction, the eccentric vortex is the most important flow phenomenon in a CFF. It is a vortical flow formed inside the impeller by the emergence of vortices shed from blades, and it is known that its location and strength are closely related to the performance of the CFF $[4,5]$. Generally, the eccentric vortex is in the close vicinity of the stabilizer, where flow 


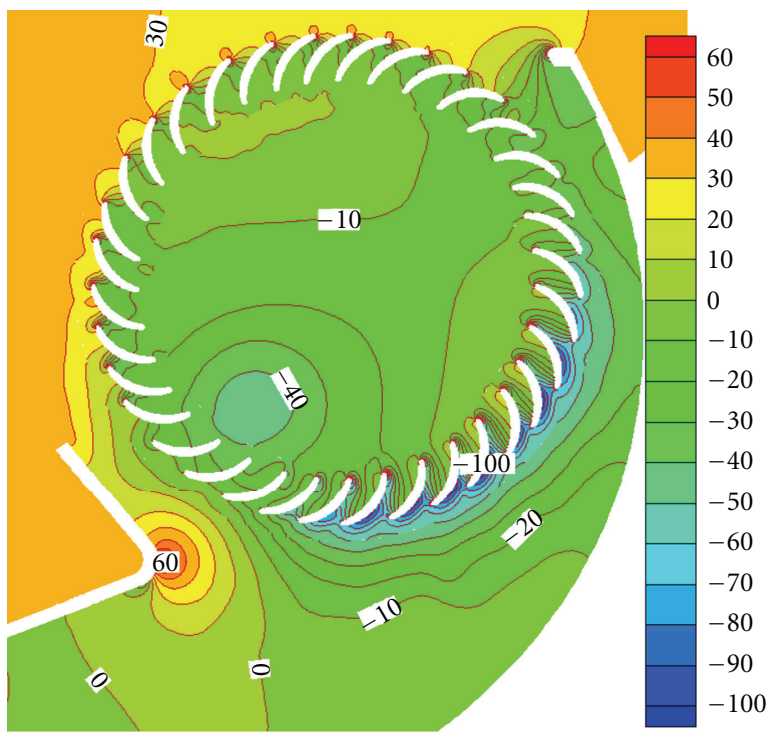

(a) Case $\mathrm{A}$

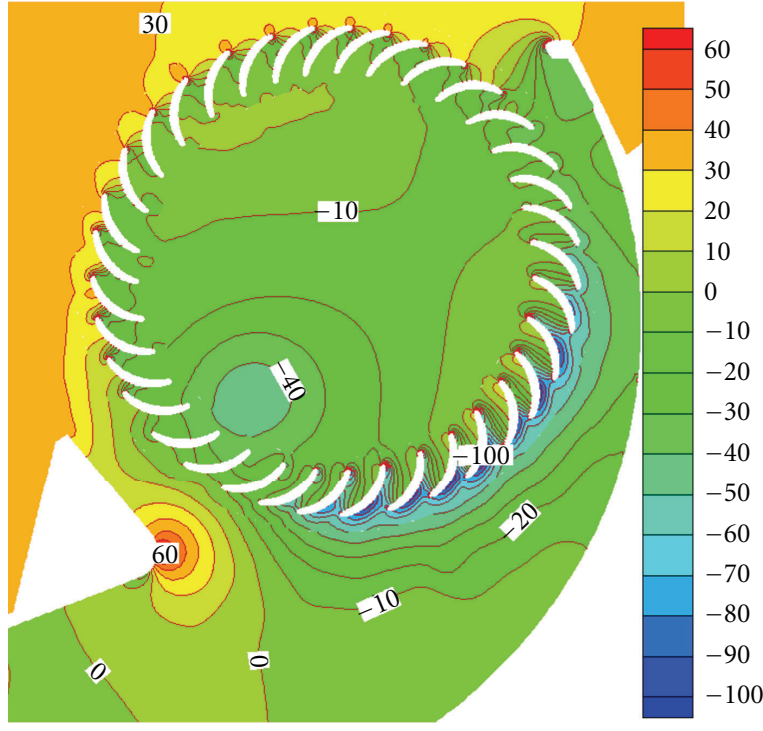

(b) Case A1

Figure 7: Comparison of calculated instantaneous static pressure contours. The contours for both cases are taken at the end of 4 rotations of the impeller from a same initial field (a) Case A. (b) Case A1.

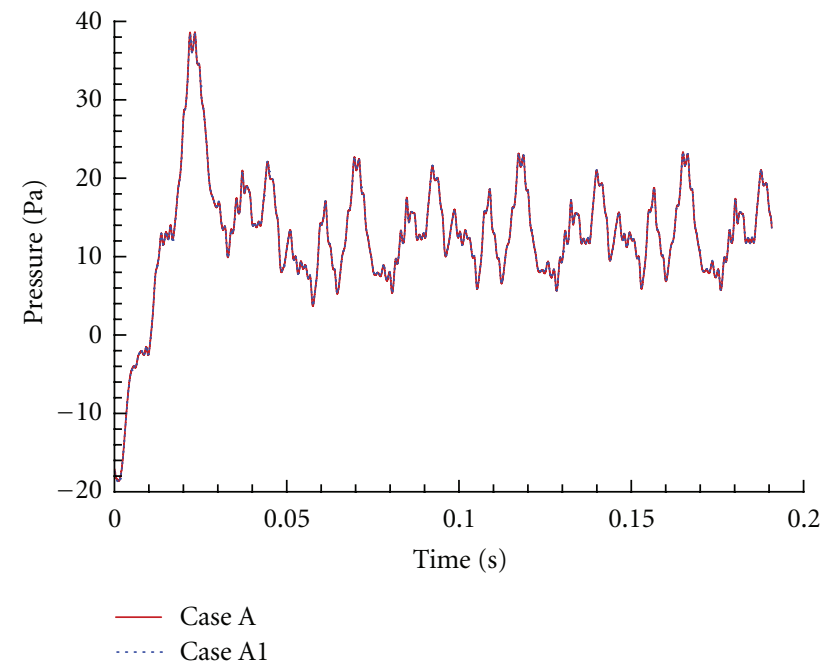

Figure 8: Comparison of time history of pressure oscillation at a monitoring point in the gap between the stabilizer and impeller. Coordinates of monitoring point are $(-0.04,-0.0375)$, while the center of impeller is at $(0,0)$.

impingement on the wall occurs. In order to examine the influences of using the porous stabilizers on the flow field, the distribution of the total pressure is compared in Figure 11. The contours shown in the figure are taken at a same instant corresponding to the oscillation of the monitored pressure in a rotation of the impeller, which will be shown later. In Figure 11, troughs of the total pressure can be observed in the eccentric vortex for each stabilizer case, respectively.

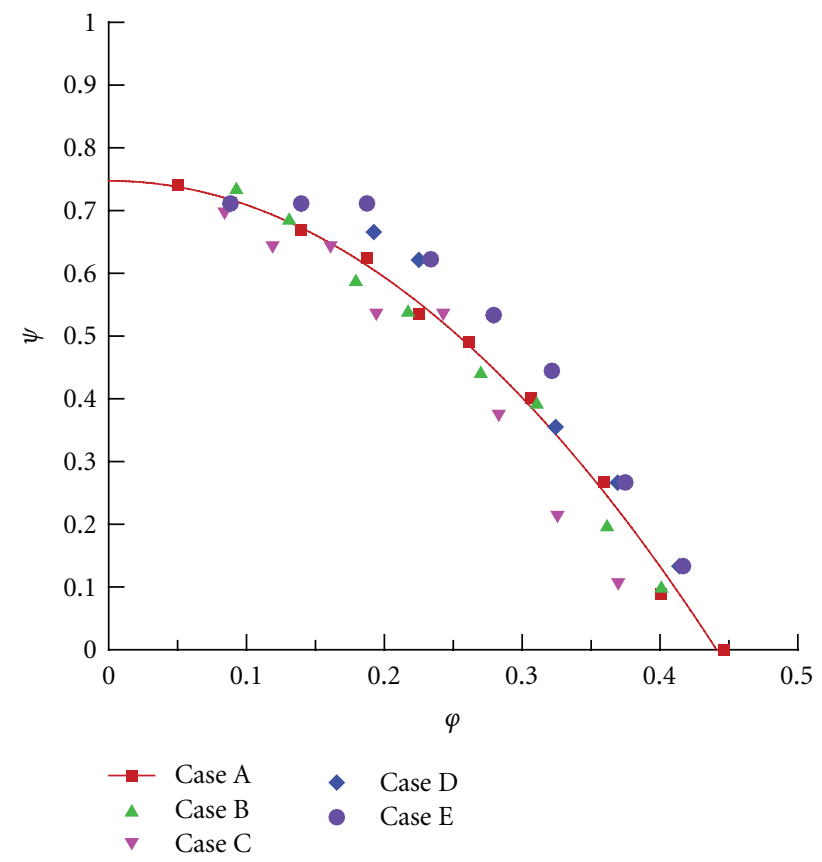

FIGURE 9: The $\psi-\varphi$ curve of the tested CFF using porous stabilizers.

Meanwhile, crests of the variable are also found near the front surface of the stabilizers. The difference between the trough and crest values in Case $\mathrm{A}$ is $110 \mathrm{~Pa}$. Compared with Case $\mathrm{A}$, such difference for Cases $\mathrm{B}, \mathrm{C}$, and $\mathrm{D}$ is $85 \mathrm{~Pa}, 90 \mathrm{~Pa}$, and $95 \mathrm{~Pa}$, respectively. This means the flow field becomes more uniform when porous stabilizers are used, which is beneficial for weakening the flow impingement on the stabilizer wall. 


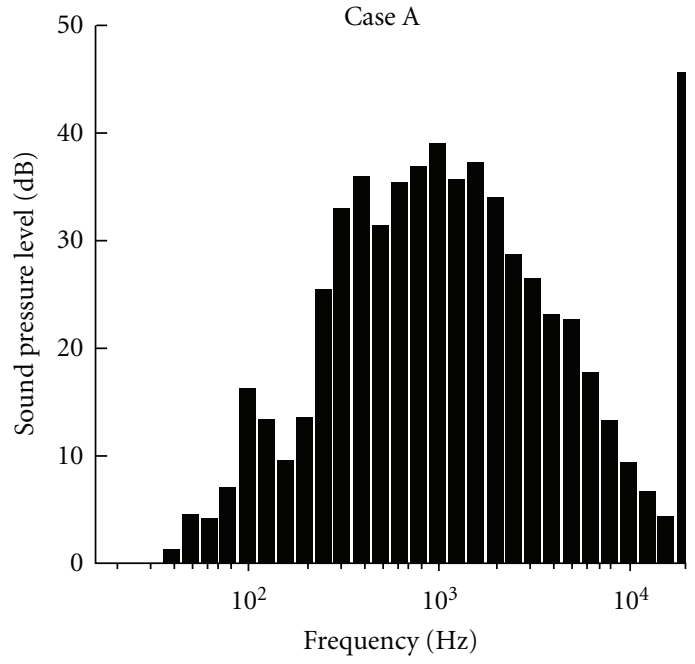

(a)

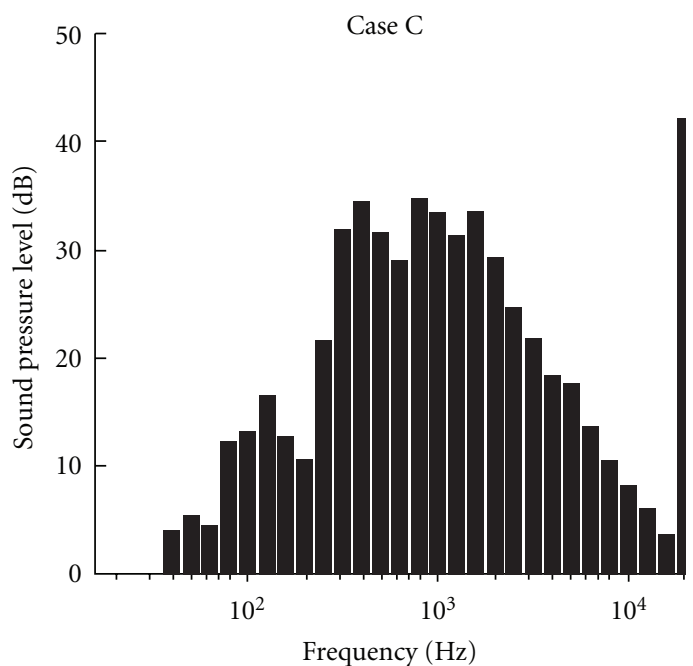

(c)

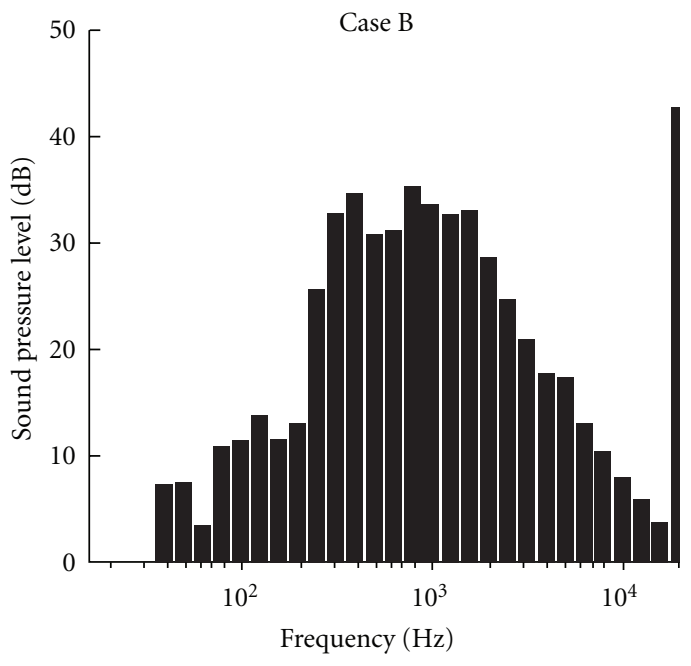

(b)

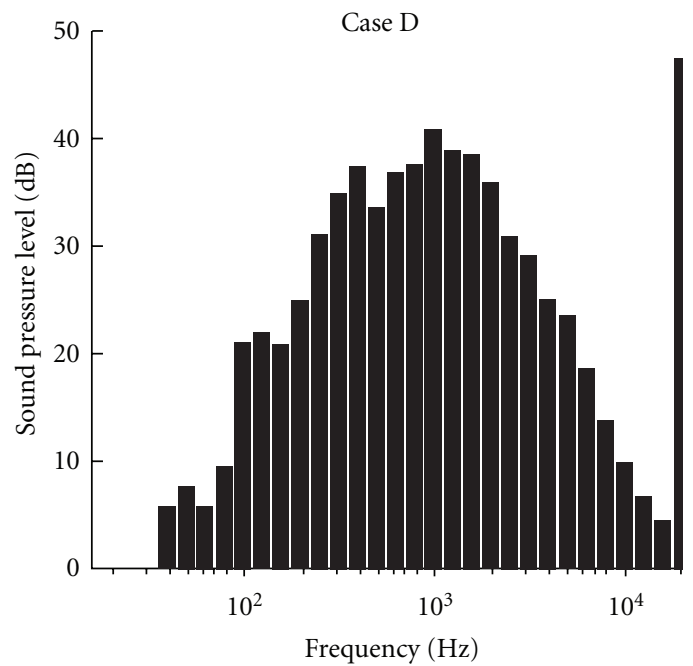

(d)

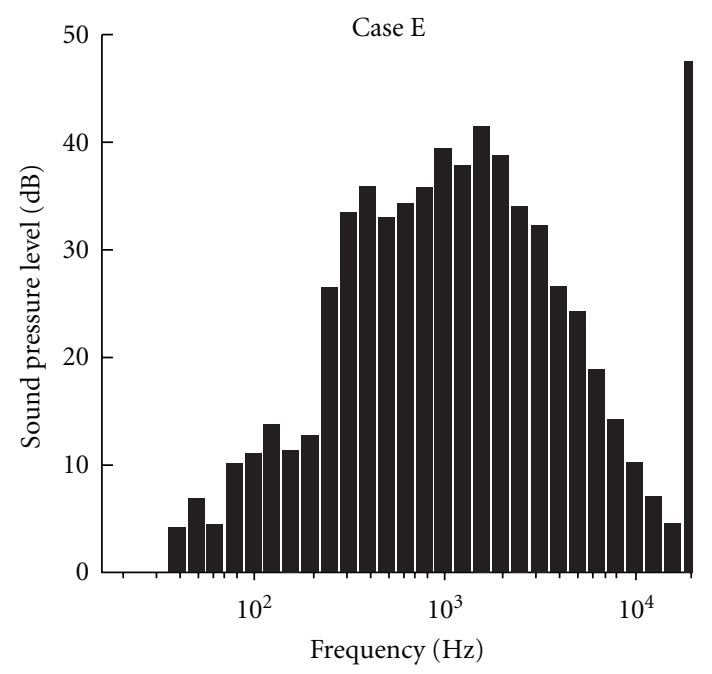

(e) 
Case A

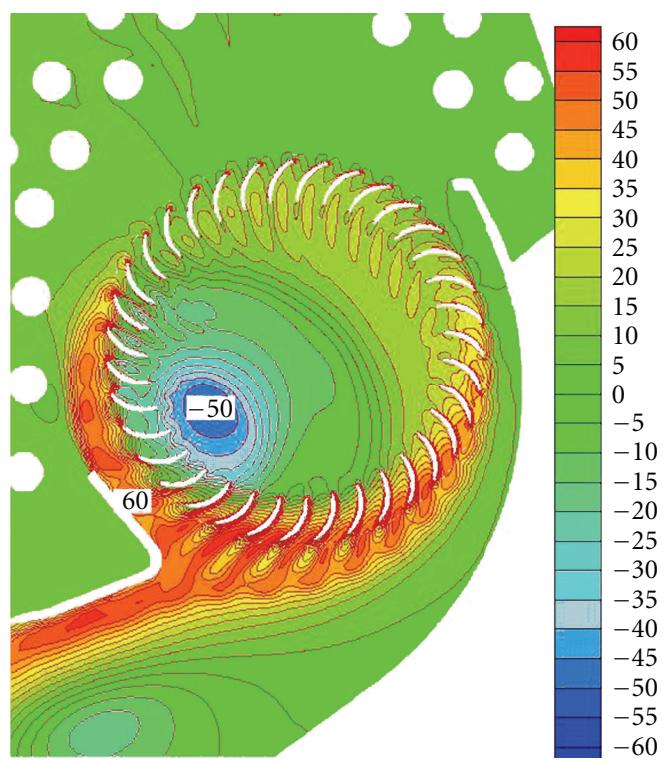

(a)

Case C

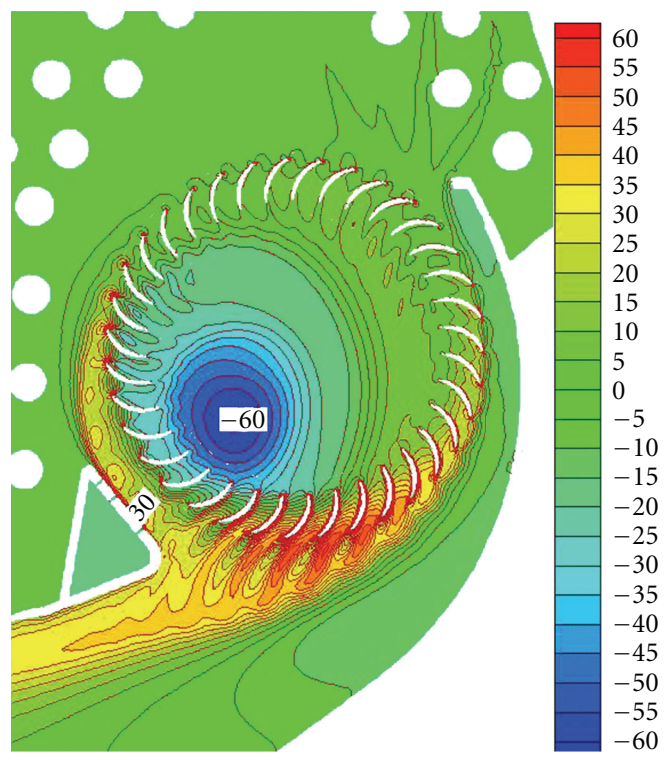

(c)
Case B

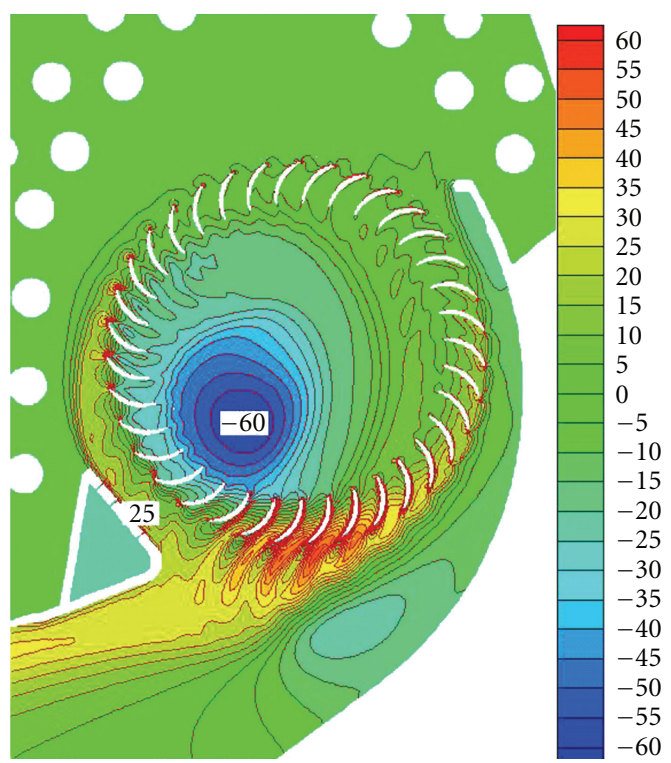

(b)

Case D

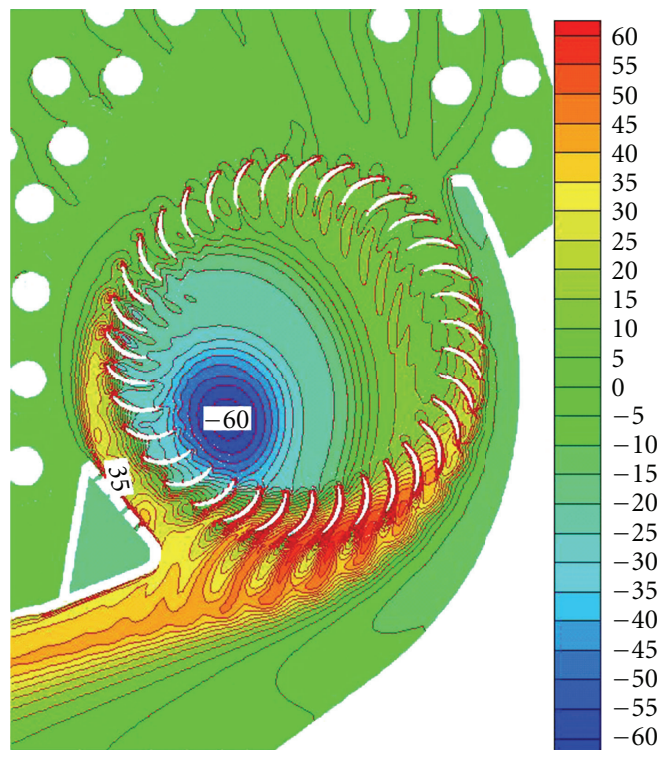

(d)

FIGURE 11: Contours of stagnant pressure for the CFF using porous stabilizers.

Figure 12 shows the instantaneous flow patterns in the eccentric vortex/stabilizer region and the associated flow in the stabilizer plenum for Case C. Because the pressures at both sides of the porous wall of the stabilizer are different, air through the CFF may move into or out of the stabilizer's plenum chamber and result in a weak flow in it. Compared with the flow traversing the fan, this flow in the stabilizer is at very low speed and is laminar. Apparently, flow transition occurs in this region, so a more sophisticated turbulence model and more elaborate simulations are needed if analysis on this creeping flow is intended in the future. The flows in the stabilizer plenum and through the porous wall are quite similar to those observed by Lai and Luo [13] in the porous cavity wall for flow and sound controlling. Although the creeping flow occupies only a negligible tiny part of mechanical energy, they are believed to play a role of weakening the flow impingements on the wall. Because of this, the amplitude of pressure oscillation in the CFF is reduced, as those shown at the monitored points in Figure 13. 


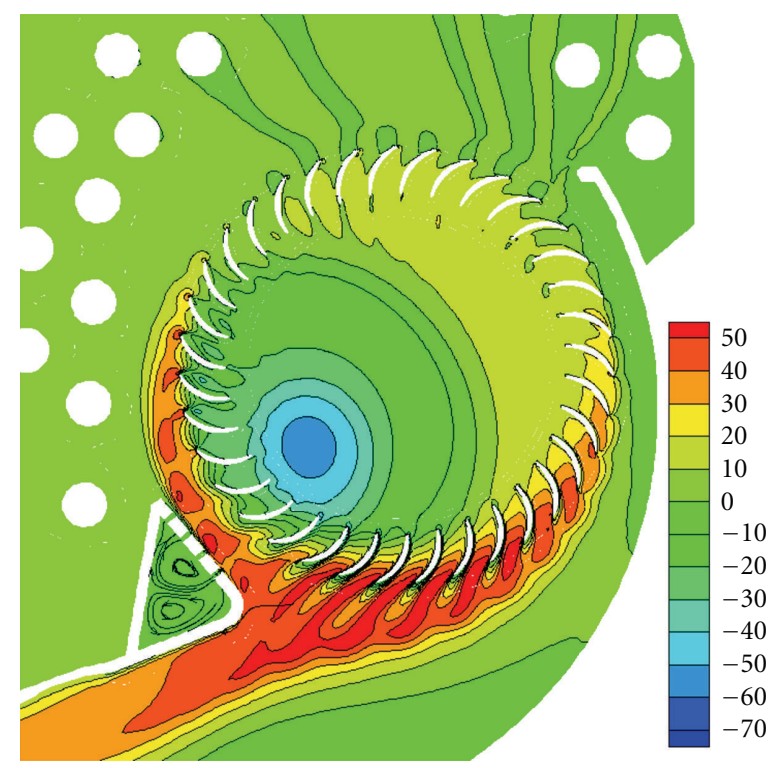

(a)

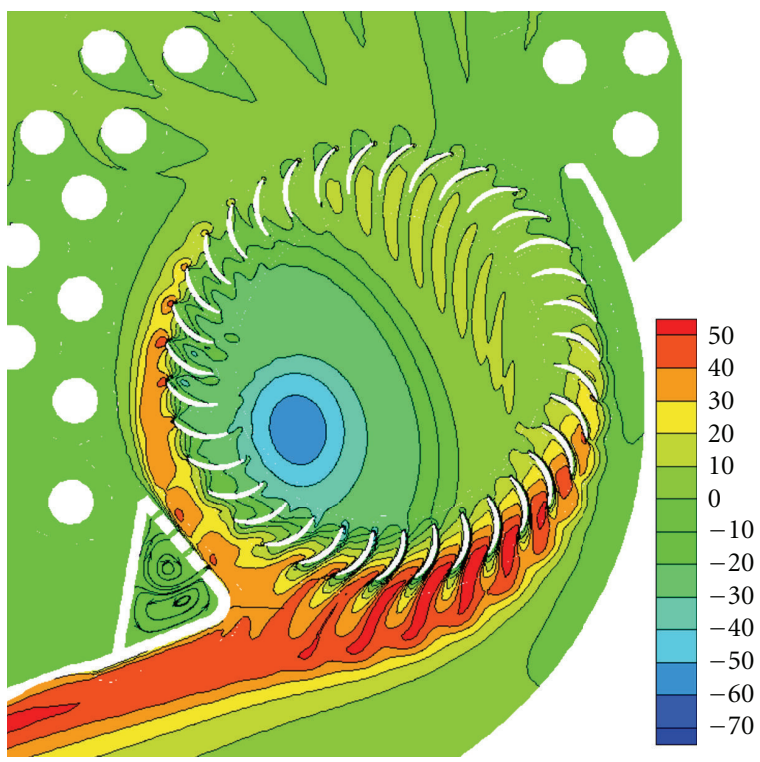

(b)

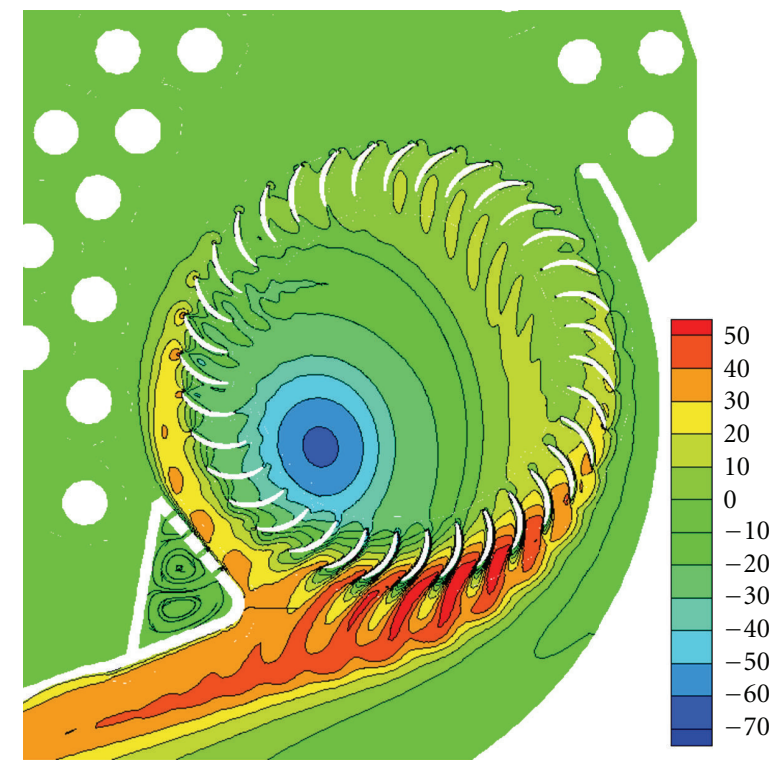

(c)

Figure 12: Instantaneous stagnant pressure for Case C. (a) at $t=t_{0}+1 / 6 T$, (b) at $t=t_{0}+1 / 2 T$, and (c) at $t=t_{0}+5 / 6 T$. $T$ is the blade passing-by period, and $t_{0}$ is the start time of a flow cycle. The unit is $\mathrm{Pa}$.

\section{Concluding Remarks}

A porous strucutre of stabilizer is proposed for attenuating the internal flow and noise of CFF. The structure consists of a porous front wall and a plenum chamber behind it. The intention is to weaken the vortical flow impingement on the wall so as to reduce the sound radiation from the CFF. Experiments and calculations are carried out for a qualitative analysis of the feasibility of such a noise-controlling method. The results show that the porous stabilizers have not produced noticeable side effects on the fan's $\psi-\varphi$ performance curve, but it does have produced important effects on the radiated noise from the fan. In the four tested porous cases, the two stabilizers with lower porosities are helpful for silencing the fan, while the other two cases with higher porosities have produced a contrary tendency. The very weak flow moving in or out of the porous wall of the stabilizers is believed to have played an important role in uniformizing the pressure in the zone of the stabilizer and the eccentric vortex, so it is helpful for weakening the vortical flow impingement on the stabilizer wall and reducing the relevant pressure oscillations. 


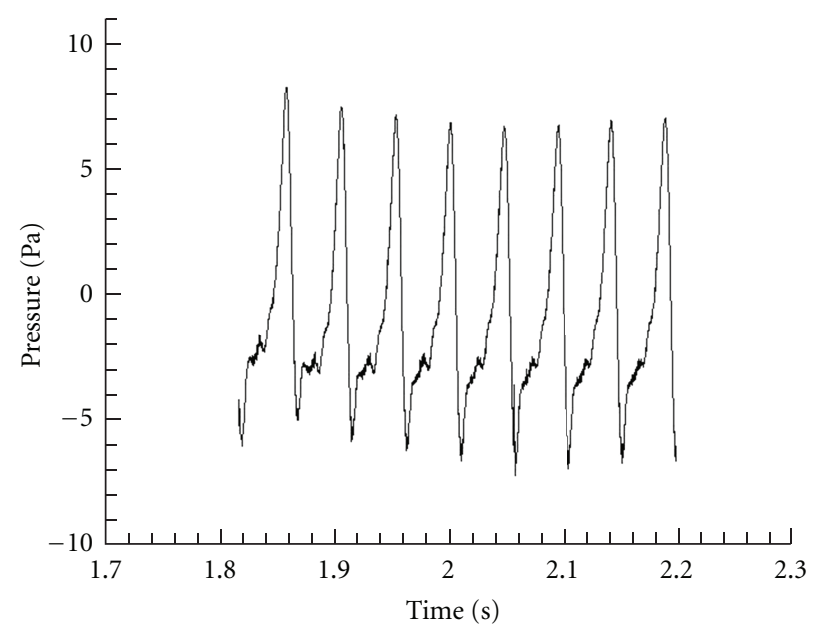

(a) Case A

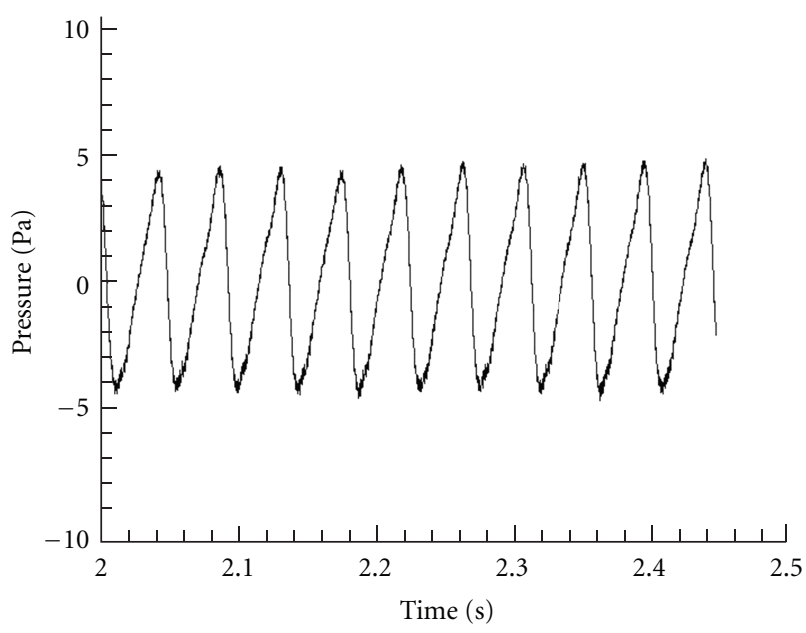

(b) Case C

FIGURE 13: Time history of the pressure at a monitored point on the surface of the stabilizer.

\section{Acknowledgments}

This work is financially supported by the NSFC under grant no. 50876031 and by Shanghai Municipal Education Commission under Grant no. 10ZZ40.

\section{References}

[1] Y. C. Shih, H. C. Hou, and H. Chiang, "On similitude of the cross flow fan in a split-type air-conditioner," Applied Thermal Engineering, vol. 28, no. 14-15, pp. 1852-1864, 2008.

[2] K. Matsuki, Y. Shinobu, A. Takushima, and S. Tanaka, "Experimental study of internal flow of a room air conditioner incorporating a cross flow fan," ASHRAE Transactions, vol. 94, no. 1, pp. 350-364, 1988.

[3] A. Takushima, Y. Shinobu, S. Tanaka, and K. Matsuki, "Flow measurement by laser doppler velocimeter in a cross-flow fan for airconditioning use," ASHRAE Transactions, vol. 96, no. 1, pp. 497-501, 1990.

[4] Y. J. Moon, Y. Cho, and H. S. Nam, "Computation of unsteady viscous flow and aeroacoustic noise of cross flow fans," Computers and Fluids, vol. 32, no. 7, pp. 995-1015, 2003.

[5] Y. Cho and Y. J. Moon, "Discrete noise prediction of variable pitch cross-flow fans by unsteady Navier-Stokes computations," ASME Journal of Fluids Engineerig, vol. 125, no. 3, pp. 543-550, 2003.

[6] I. B. Eck, Fans: Design and Operation of Centrifugal, Axial Flow and Cross-flow Fans, Pergamon Press, 1952.

[7] H. M. Koo, "Discrete frequency noise reduction of the crossflow fan of the split type room air-conditioners using the skewed stabilizers," JSME International Journal Series C, vol. 43, no. 1, pp. 104-109, 2000.

[8] H. M. Koo, "An experimental study of the noise and the performance of cross-flow fans in room air-conditioning systems," Noise Control Engineering Journal, vol. 48, no. 2, pp. 41-47, 2000.

[9] A. Toffolo, A. Lazzaretto, and A. D. Martegani, "Crossflow fan design guidelines for multi-objective performance optimization," Proceedings of the Institution of Mechanical Engineers, Part A, vol. 218, no. 1, pp. 33-42, 2004.
[10] G. L. Tsai, T. H. Tu, T. C. Li, and K. H. Wang, "Flow style investigation and noise reduction of a cross-flow fan with varied rotor-skew-angle rotor," JSME International Journal Series B, vol. 49, no. 3, pp. 695-704, 2006.

[11] P. Thiede, P. Krogmann, and E. Stanewsky, Active and Passive Shock/Boundary Layer Interaction Control on Supercritical Airfoils, AGARD CP-365, 1984.

[12] F. J. Wilcox, "Passive venting system for modifying cavity flowfields at supersonic speeds," AIAA journal, vol. 26, no. 3, pp. 374-376, 1988.

[13] H. Lai and K. H. Luo, "Large-Eddy simulation and control of cavity aeroacoustics using porous media inserts," Flow, Turbulence and Combustion, vol. 80, pp. 375-391, 2008.

[14] Y. N. Jeng and U. J. Payne, "Numerical study of a supersonic open cavity flow and pressure oscillation control," Journal of Aircraft, vol. 32, no. 2, pp. 363-369, 1995.

[15] I. Kim and N. Chokani, "Navier-Stokes study of supersonic cavity flowfield with passive control," Journal of Aircraft, vol. 29, no. 2, pp. 217-223, 1992.

[16] I. J. Hughes and A. P. Dowling, "Absorption of sound by perforated linings," Journal of Fluid Mechanics, vol. 218, pp. 299-335, 1990.

[17] FLUENT V.6.1, User's guide, FLUENT Inc., USA, 2003.

[18] B. E. Launder and D. B. Spalding, "The numerical computation of turbulent flows," Computer Methods in Applied Mechanics and Engineering, vol. 3, no. 2, pp. 269-289, 1974.

[19] S. V. Patankar, Numerical Heat Transfer and Fluid Flow, Hemisphere Publishing Corporation, New York, NY, USA, 1980. 

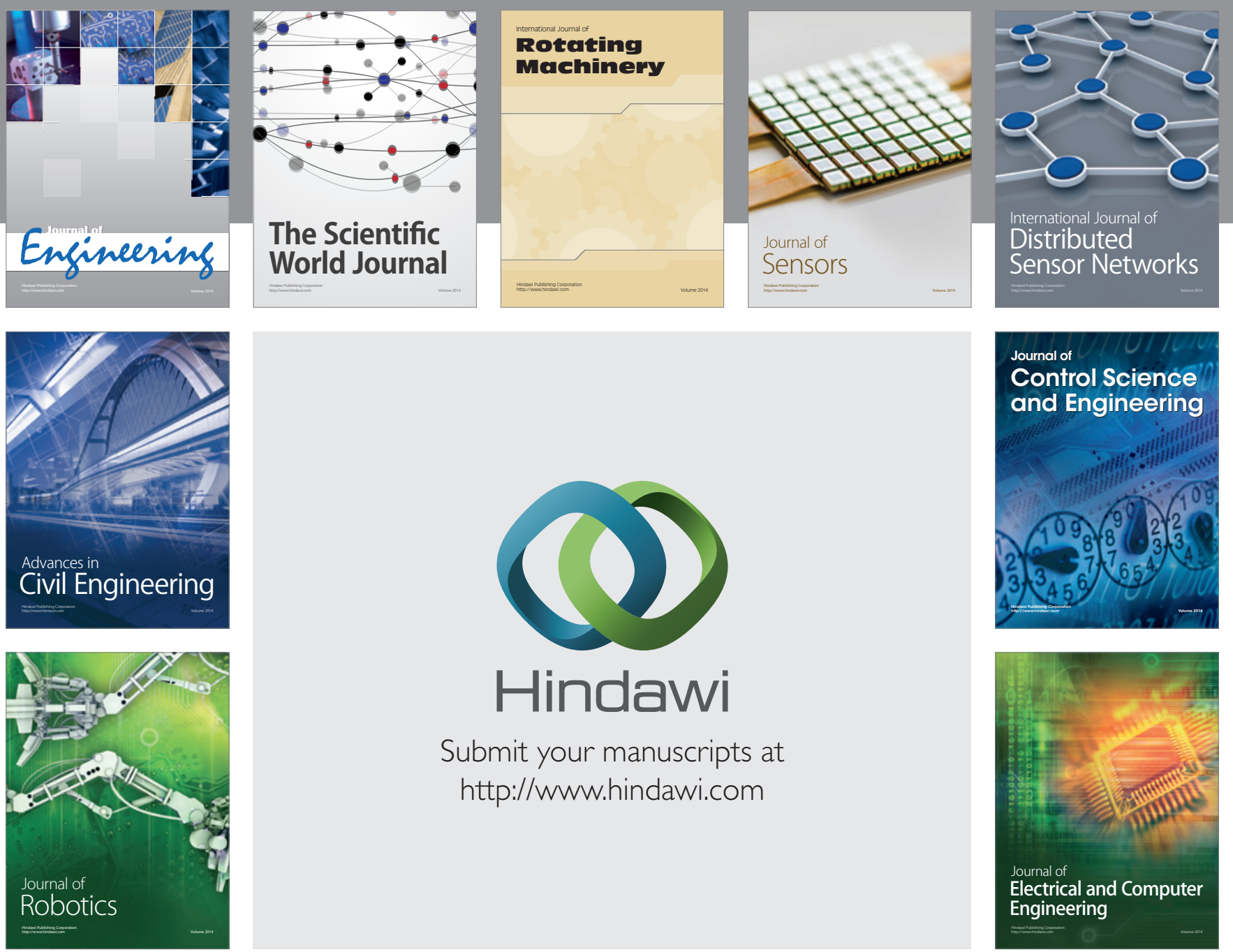

Submit your manuscripts at

http://www.hindawi.com
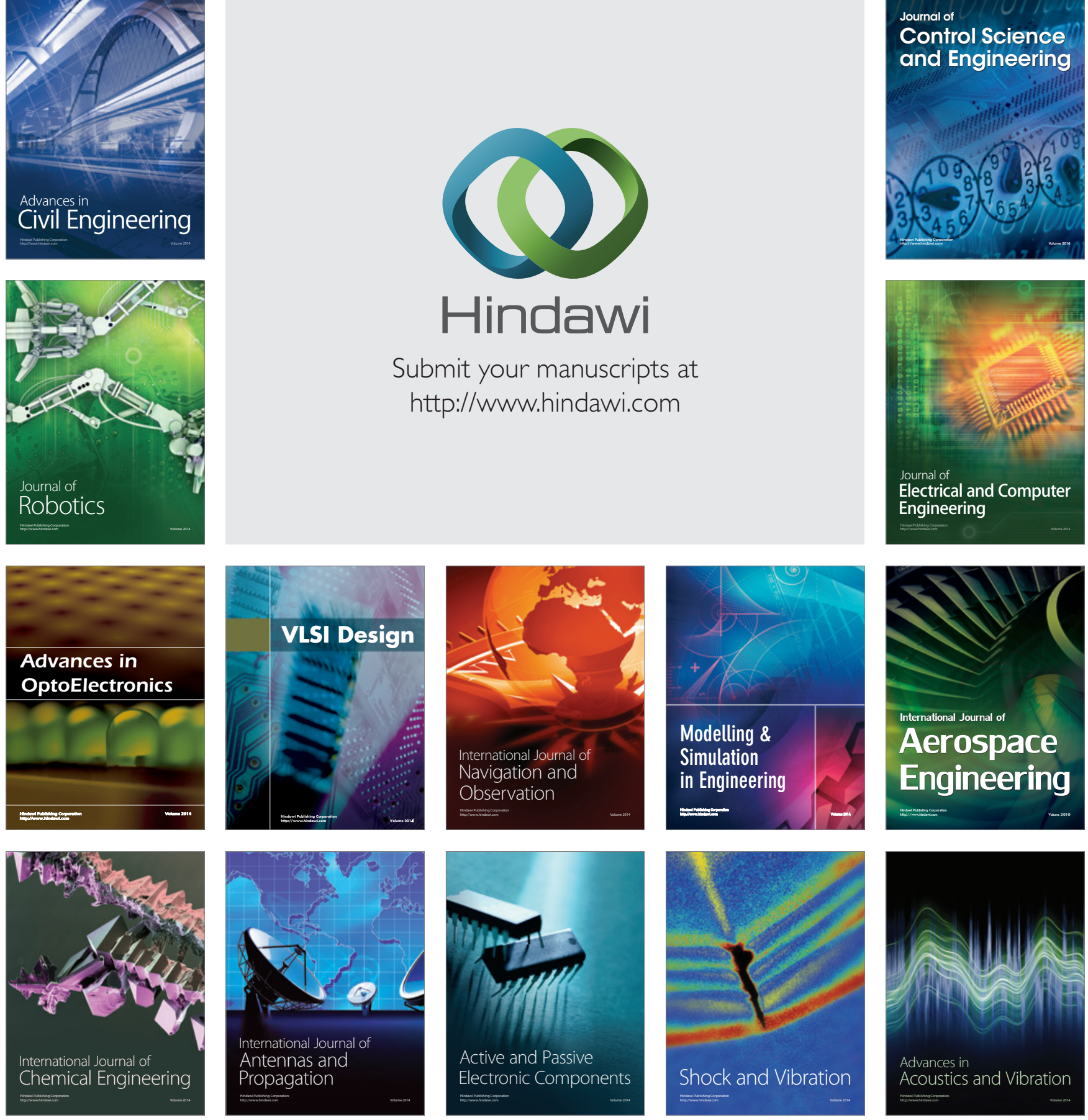